\title{
Immunohistochemical Study of Extracellular Matrices and Elastic Fibers in a Human Sternoclavicular Joint
}

\author{
By
Kazuyuki SHIMADA*, Naotoshi TAKESHIGE, Hiroshi MORIYAMA*, Yasushi MIYAUCHI*, Shigetaka SHIMADA* and Etsuo FUJIMAKI \\ *Department of Anatomy and Department of Orthopedic Surgery, Showa University School of Medicine, \\ 5-8, Hatanodai 1, Shinagawa-ku, Tokyo 142, Japan
}

- Received for Publication, September, 1997 -

\begin{abstract}
Key Words: Sternoclavicular joint, Elastic fibers, Oxytalan fibers, Immunohistochemistry, Extracellular matrix
Summary: In this study, we clarified the distribution of elastic and oxytalan fibers in a human sternoclavicular joint (SCJ) using a color image system and in extracellular matrices using immunoperoxidase staining. Fine elastic fibers (EFs) were scattered in the fibrous layer of the sternoclavicular disk. This articular disk was composed of a collagenous bundle on the sternum side of the articular disk in the SCJ and cellular components including connective tissue on the clavicular side of the articular disk. The thickness of the disk gradually increased from the inferior to superior portion. Collagen fibers type I, III and V and other extracellular matrices (ECMs) were detected in the hypertrophic zone in the clavicular and sternum side of the SCJ and in the connective tissue of the articulatio condylar. On the cervical surface of the articular disk, cellular activity was higher than on the sternum surface.
\end{abstract}

The human sternoclavicular joint (SCJ) resembles the temporomandibular joint (TMJ) from the viewpoint of having a cartilaginous head and an articular disk, but details of the morphology and cellular activity of these joints on the immunohistochemical level are unknown. Moreover, some investigators have reported reconstructing the TMJ using sternoclavicular and costochondral grafts (Dingman and Grabb, 1964; Wolford et al., 1994; MacIntosh and Henny, 1977). The success rate of reconstruction is higher than that of use of synthetic implants (Wolford et al., 1994). Ellis and Carlson (1986) also indicated similarities between the SCJ and TMJ in the monkey. However, the cellular activity, development and functional property of the SCJ have not yet been determined. In particular, the ECMs are functionally and developmentally associated with cell activity (Takahashi et al., 1995; Mizoguchi et al., 1997). Therefore, we clarified the distributions of the EF and ECM components in the human SCJ and compared them with those in the human TMJ.

\section{Material and Methods}

\section{Preparation of tissue}

Adult humans with non-gross pathological changes were selected at autopsy from donors (twenty-four males aged 27 to 70 years) for a histochemical analysis at microscopic levels. The sternoclavicular joints were quickly removed and immediately fixed in a $3.7 \%$ solution of formaldehyde for one day at $4^{\circ} \mathrm{C}$. After washing them in running water, they were dehydrated in a graded series of ethanol (50, 70, 90 and $95 \%$ and absolute) followed by paraffin embedding. Paraffin blocks were cut into $3-\mu \mathrm{m}$-thick frontal serial sections.

Staining and analysis of elastic and oxytalan fibers

Sections were stained by the following methods:

(1) Elastica-van Gieson staining was performed to demonstrate the locations of elastic fibers.

(2) Elastica-van Gieson staining after incubation in a preoxidizing solution ( $\mathrm{pH} 1.5$ ) containing $0.3 \%$ $\mathrm{KMnO}_{4}$ and $0.3 \% \mathrm{H}_{2} \mathrm{SO}_{4}$ was performed to visualize oxytalan fibers (modified version of the method

Address correspondence to Kazuyuki Shimada, D.D.S., Ph.D., Department of Anatomy, Showa University School of Medicine, 5-8, Hatanodai 1, Shinagawa-ku, Tokyo 142, Japan.

This work was supported by Grant No. 08671692 from the Ministry of Education, Science and Culture of Japan. 
of Fullmer, 1959). Serial sections were observed using a real color image system (Swallo II, Interquest, Osaka, Japan). This system was configured by a color image measurement system using a combination of a video camera (TK-870, Victor JVC, Yokohama, Japan), digital VX color video monitor (Digital PC, DEC, Tokyo, Japan) and a light microscope (Vanox-S, Olympus, Tokyo, Japan). Five measurement areas (each area: $10,000 \mu^{2}$ ) were selected in the articular disk of the SCJ.

\section{Immunoperoxidase staining}

Primary antibodies

Rabbit monoclonal antibodies directed against rat tail tendon collagen types I, III and V (LSL Co., Tokyo, Japan), bovine blood plasma fibronectin (LSL Co., Tokyo, Japan), human fibroblast tenascin (BIOHIT OY, Helsinki, Finland) and mouse EHS sarcoma laminin (LSL Co., Tokyo, Japan) were used.

\section{Immunohistochemistry}

After demineralization with ethylenediaminetetraacetic acid $(0.3 \mathrm{ml} / \mathrm{L})$ overnight at $4{ }^{\circ} \mathrm{C}$ and washing in running water, the specimens were dehydrated in absolute ethanol, followed by paraffin embedding. Paraffin blocks were cut into $10 \mu \mathrm{m}$ thick sagittal serial sections. These specimens were examined using six monoclonal antibodies that were specific for the connective tissue macromolecules. The sections were treated with methanol containing $0.5 \% \mathrm{H}_{2} \mathrm{O}_{2}$ for $30 \mathrm{~min}$. to inhibit endogenous peroxidase activity, and washed for $10 \mathrm{~min}$. in three changes of phosphate-buffered saline (PBS) (pH 7.2). They were subsequently incubated in a mixture of normal goat serum and PBS containing $20 \mathrm{mg} / \mathrm{ml}$ bovine serum albumin for $30 \mathrm{~min}$. at room temperature in order to prevent non-specific adsorption of the antibodies during later stages of the procedure. After separate incubations with secondary antibodies against primary antibodies, the sections were washed in PBS. After incubation with biotinylated antibody against rabbit IgG (Ctastain Elite ABC Kit, Vector Laboratories Burlingame, CA, USA), the specimens were washed in PBS, and finally incubated in a biotin-horseradish peroxidase complex (Elite ABC Kit, Vector Laboratories Burlingame, CA, USA). After washing them in running water, they were incubated with $0.02 \% \mathrm{H}_{2} \mathrm{O}_{2}$ and $0.1 \%$ diaminobenzidine tetrahydrochloride (DAB, $0.1 \mathrm{M}$ Tris buffer, $\mathrm{pH}$ 7.2). Sections were mounted with Bioleit (Kokenn, Tokyo, Japan), and observed under a light microscope (Vanox-S, Olympus Co., Tokyo) (Fig. 1).

\section{Results}

\section{Distribution of EF in SCJ as determined by \\ Elastica-van Gieson staining}

EFs were located in the specimens in the fibrous layer of the articular disk of the SCJ. Numerous collagenous bundles in the articular disk were arranged straight from the inferior to the medial region and they mostly curved in the superior region of the disk. In the superior region of the sternum and the clavicular surface of the disk, the EFs were scattered among collagen complex fibers. In the medial and inferior regions of the sternum surface of the disk, the EFs were regularly arranged along the fibrous bundles. The EFs were not found in the medial and inferior regions of the clavicular surface (Fig. 2a, 2b). A few EFs were arranged irregularly in the superior region of the clavicular surface of the disk (Fig. 2C). The thickness of the disk increased from the inferior to the superior region. Numerous nerves and vessels were observed in the proximal region of the disk and were associated with collagenous bundles originating from the proximal joint capsule (Fig. 2).

\section{Calculation of the ratios of EFs and oxytalan fibers} [OFs] to all fibers in the articular disk

In six points of the articular disk (see Fig. 1), the ratios of EFs and OFs to all fibers per measurement area (each area: $10,000 \mu \mathrm{m}^{2}$ ) were calculated using a combination of a color image system and light microscope. There was a low percentage of EFs in the disk, and the ratio of the EFs to all fibers decreased from the superior to the medial region (varying from $0.3 \pm 0.02$ to $0.1 \pm 0.01 \%$ ). This tendency was observed for each surface region of the disk. Comparing EFs with OFs, the percentage of OFs (varying from $0.2 \pm 0.01$ to $0.09 \pm 0.008 \%$ ) was lower than that of EFs in each region.

\section{Immunohistochemical observations}

Collagen types I, III, and V, laminin, tenascin and fibronectin were all detected in the articular disk of the SCJ. Collagen type I was mainly found in the inferior region of the SCJ. Regarding collagen type III there were weak reactions in the articular disk. Fibronectin and tenascin were present from the medial to the inferior region in the SCJ. In each region of the sternum side of the SCJ, fibronectin and tenascin were found. In contrast, collagen types III and V and laminin were found in each region of the clavicular side of the SCJ (Table 1) (Figs. 3, 4). 
Table 1. Distribution and Intensity of the Immunoperoxidase Reaction Specific for Collagen types I, III, V and Proteins of the Extracellular Matrix in Human Sternoclavicular Joint

\begin{tabular}{rcccccc}
\hline Points & $\begin{array}{c}\text { Collagen } \\
\text { type I }\end{array}$ & $\begin{array}{c}\text { Collagen } \\
\text { type III }\end{array}$ & $\begin{array}{c}\text { Collagen } \\
\text { type V }\end{array}$ & Fibronectin & Tenascin & Laminin \\
\hline 1 & \pm & \pm & + & + & + & + \\
2 & + & \pm & + & + & + & + \\
3 & \pm & + & ++ & + & + & + \\
4 & + & + & ++ & + & + & + \\
5 & + & + & + & + & + & + \\
6 & + & \pm & \pm & \pm & \pm & \pm \\
7 & \pm & \pm & \pm & + & + & \pm \\
8 & \pm & \pm & \pm & + & + & \pm \\
9 & + & \pm & + & + & + & \pm \\
10 & + & + & + & + & + & + \\
11 & \pm & + & + & + & + & + \\
12 & + & + & + & + & \pm & + \\
13 & \pm & + & + & + & + & + \\
14 & + & + & + & + & & + \\
\hline
\end{tabular}

Points 1-14 are indicated in Fig. 1b.

++ : strong reaction, + : moderate reaction, \pm : weak reaction, - : no reaction.

\section{Discussion}

Our color analysis system can be used for analyzing sections histochemically stained using a method modified from Fullmer and Lillie (1958), and to differentiate EF from the collagen fiber system. Moreover, the distribution of OFs and EFs from the superior to the inferior region of the human articular disk of the SCJ provides detailed information regarding morphological features. OFs are composed of elastic, pre-elastic and other fibers (Fullmer, 1960). The percentages of OFs in all measurement areas were higher than those of EFs. OFs may transform into EFs in this region. Comparing the human articular disk of the TMJ with that of the SCJ there are no EFs in that of the TMJ. EFs were only found in TMJ meniscus (Griffin and Sharpe, 1962). However, in the posterior and inferior regions of the articular disk of the mouse TMJ, EFs were found (Frommer and Monroe, 1966). These different distributions of EFs may be an indication of the functional properties of the EFs and how they change their forms for functional adaptation. Therefore, a sternoclavical graft seems to be possible for TMJ reconstruction about their adaptation.

Collagen type III was only found in the articular disk. In general, matrix components include collagen type III which contributes to bone formation (Keene et al., 1991) and pre-collagen type III is found in remodeled bone (Becker et al., 1986). Aigner et al., (1993) reported that in the superficial and upper middle layers of the osteoarthitic carti- lage, chondrocyte clusters and single chondrocytes were identified expressing collagen type III as well as type II, but not collagen type I. The expression of the cytoplasmic alpha 1 (III) mRNA identified by in situ hybridization was consistent with the immunohistochemical localization of the extracellular collagen type III in this area. In noncartilagenous tissue, collagen type III is expressed in vivo and in vitro for the most part in association with collagen type I, although in different ratios (Hill et al., 1984; Layman et al., 1977). Therefore, the presence of type III collagen is associated with the cellular activity in the intercellular substance of subperiosteal bone. In our study, collagen type III was found mainly in the cartilagenous area of the cervical and sternum side of the SCJ. In contrast, in the human pathological TMJ, collagen types II and III were found in the repaired tissue and hypertrophic zone of condylar cartilage (Salo and Raustia, 1995). Differences in the distribution of ECMs indicate differences in cellular activity with respect to various functional properties. These reports show the importance of the appearance and distribution of the ECMs in the morphological description of the articular joint. The hypertrophic zone of collagen fibers was detected in the clavicular and sternum side of the SCJ. In particular, collagen type I was found in the inferior region of the articular disk. Fibronectin, tenascin and laminin were found in the sternum side of the SCJ. This distribution of the ECMs suggested that cellular properties differ with the stage of remodeling of the bone. Takahashi et al. (1995) and Mizoguchi et al. (1997) suggested that 
collagen fibers were found in the posterior region of the mandibular condylar which indicated high cellular activity during remodeling. In the hypertrophic zone of the clavicular and sternum side of the SCJ, there may also be a high level of cell activity to facilitate the remodeling of the bone matrix. The presence of ECMs is one of the most important factors for remodeling and modeling the bone matrix. Therefore, there is a possibility of a change in the distribution of ECMs after implanting the SCJ in the TMJ.

\section{References}

1) Aigner T, Bertlin W, Stöss $H$, Weseloh $G$ and Von der Mark K. Independent expression of febrile-forming collagens I, II, and III in chondrocytes of human osteoarthritic cartilage. J Clin Invest 1993; 91:829-837.

2) Becker J, Schuppan D, Hahn EG, Albert G and Reichart $P$. The immunohistochemical distribution of collagens type IV, V, VI and of laminin in the human oral mucosa. Arch Oral Biol 1986; 31:179-196.

3) Dingman RO and Grabb WG. Reconstruction of both mandibular condyles with metatarsal bone grafts. Plast Reconstr Surg 1964; 34:441-451.

4) Ellis E III and Carlson DS. Histologic comparison of the costochondral, sternoclavicular, and temporomandibular joints during growth in macaca mulatta. J Oral Maxillofac Surg 1986; 44:312-321.

5) Fullmer HM and Lillie RD. The oxytalan fiber; a previously undescribed connective tissue fiber. J Histochem Cytochem 1958; 6:425-430.

6) Fullmer HM. Observation on the development of oxytalan fibers in the periodontium of man. J Dent Res 1959; 38:510-518.

7) Fullmer HM. A comparative histochemical study of elastic, pre-elastic and oxytalan connective tissue fibers. J Histo- chem Cytochem 1960; 8:290-295.

8) Frommer $J$ and Monroe CY. Development and distribution of elastic fibers in the mandibular joint of the mouse: A comparison of fetal, suckling, juvenile and adult stages. Anat Rec 1966; 156:333-346.

9) Griffin CJ and Sharpe CJ. Distribution of elastic tissue in the human temporomandibular meniscus especially in respect to compression areas. Aust Dent J 1962; 7:72-78.

10) Hill RJ and Haper E. Quantitation of types I and III collagens in human tissue samples and cell culture by bromide peptide analysis. Anal Biochem 1984; 141:83-93.

11) Keene DR, Sakai LY and Burgeson RE. Human bone contains type III collagen, type IV collagen and fibrillin: type III collagen is present on specific fibers that may mediate attachment of tendons, ligaments and periosteum to calcified bone cortex. J Histochem Cytochem 1991; 39:59-69.

12) Layman D, Epstein E, Dodson R and Titus J. Biosynthesis of type I and III collagens by cultured smooth muscle cell from human aorta. Proc Natl Acad Sci USA 1977; 74:671675.

13) MacIntosh RB and Henny FA. A spectrum of application of autogenous costochondral grafts. J Oral Maxillofac Surg 1977; 5:257-267.

14) Mizoguchi I, Takahashi I, Sasano $Y$, Kagayama $M$ and Mitani H. Localization of type I, II and III collagen and glycosaminoglycans in the mandibular condyle of growing monkeys: an immunohistochemical study. Anat Embryol 1997; 195:127-135.

15) Salo LA and Raustia AM. Type II and type III collagen in mandibular condylar cartilage of patients with temporomandibular joint pathology. J Oral Maxllofac Surg 1995; 53:39-44.

16) Takahashi I, Mizoguchi I, Nakamura $M$, Kagayama $M$ and Mitani $\mathbf{H}$. Effects of lateral pterygoid muscle hyperactivity on differentiation of mandibular condyles in rats. Anat Rec 1995; 241:328-336.

17) Wolford LM, Cottrell DA and Henry C. Sternoclavicular grafts for temporomandibular joint reconstruction. J Oral Maxllofac Surg 1994; 52:119-128.

\section{Explanation of Figures}

\section{Plate I}

Fig. 1. Frontal section of a human sternoclavicular joint subjected to Elastica-van Gieson staining (a, $\times 5)$. Schematic of the areas (1-14 points) subjected to immunohistochemical analyses (b). C: clavicle; S: sternum. 

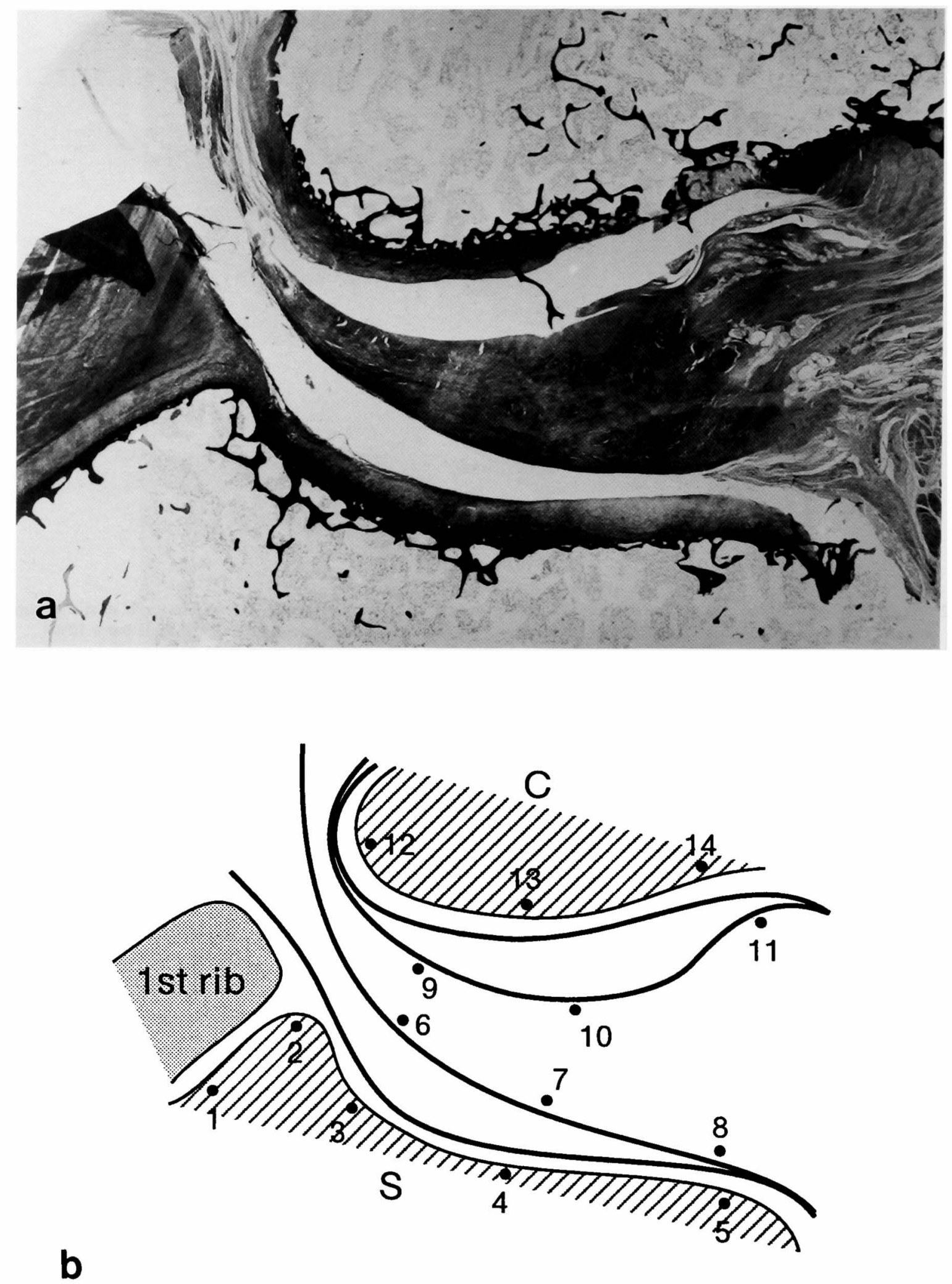
Plate II

Fig. 2. Light micrographs of sections of an articular disk subjected to Elastica-van Gieson staining (a: inferior; b: medial; c: superior regions of the clavicular surface of the disk). Elastic fibers cut vertically are indicated by an arrow. Scale bar $=10 \mu \mathrm{m}$. 

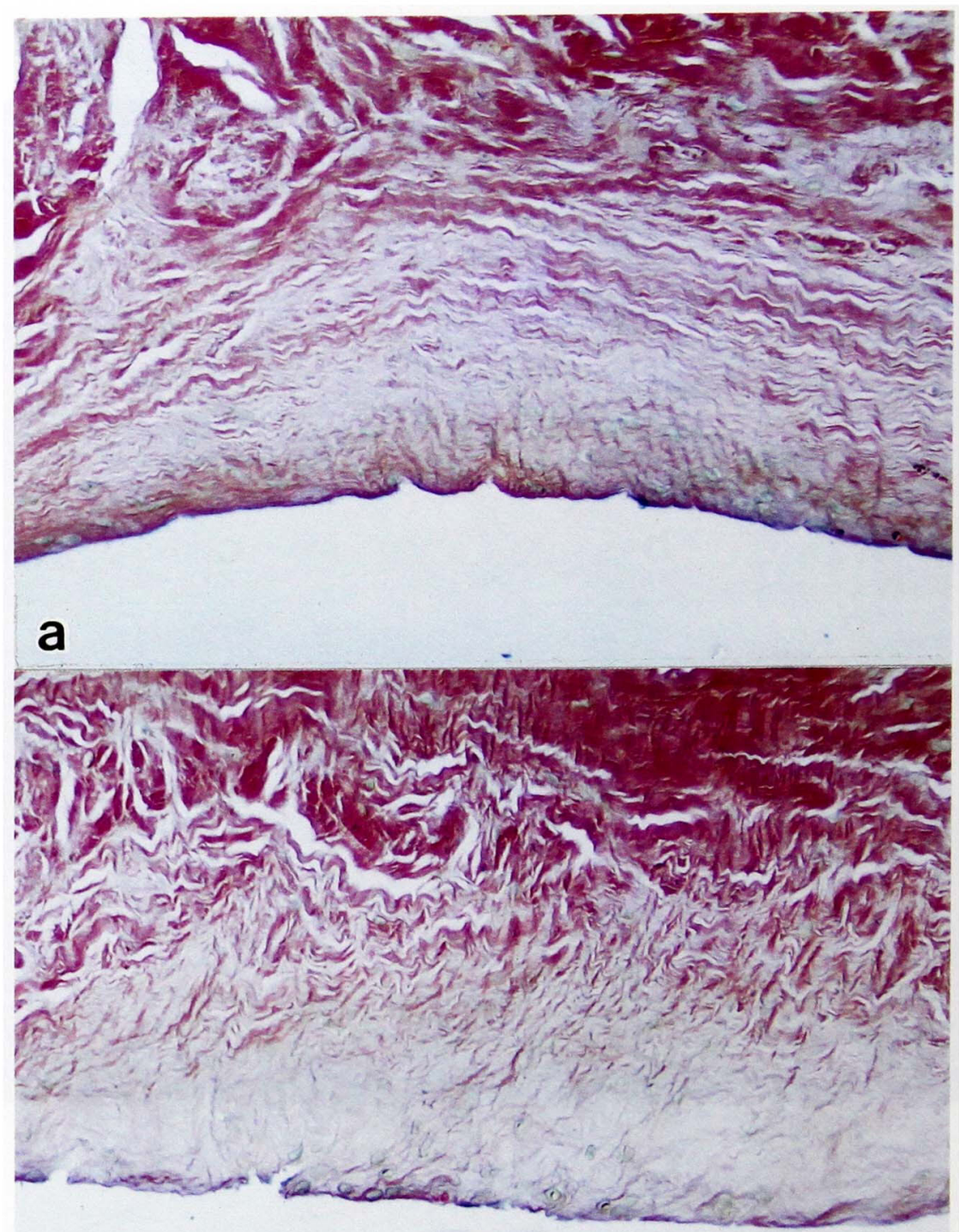

\section{b}

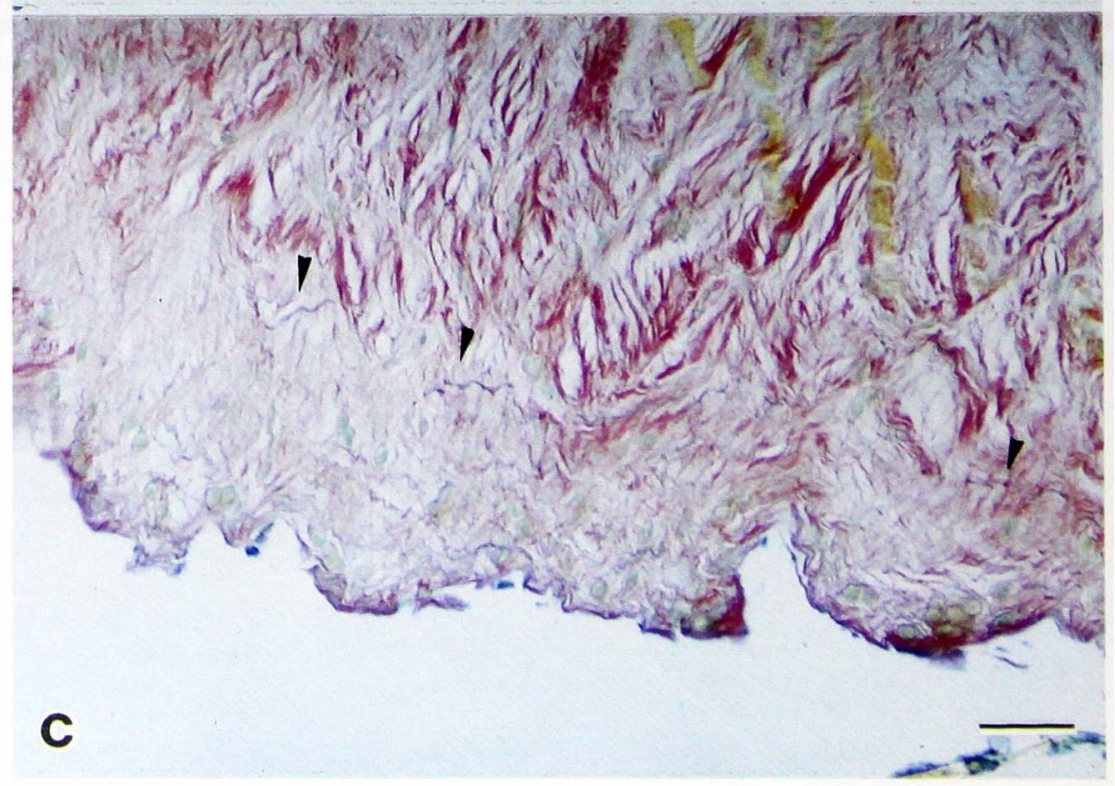


Plate III

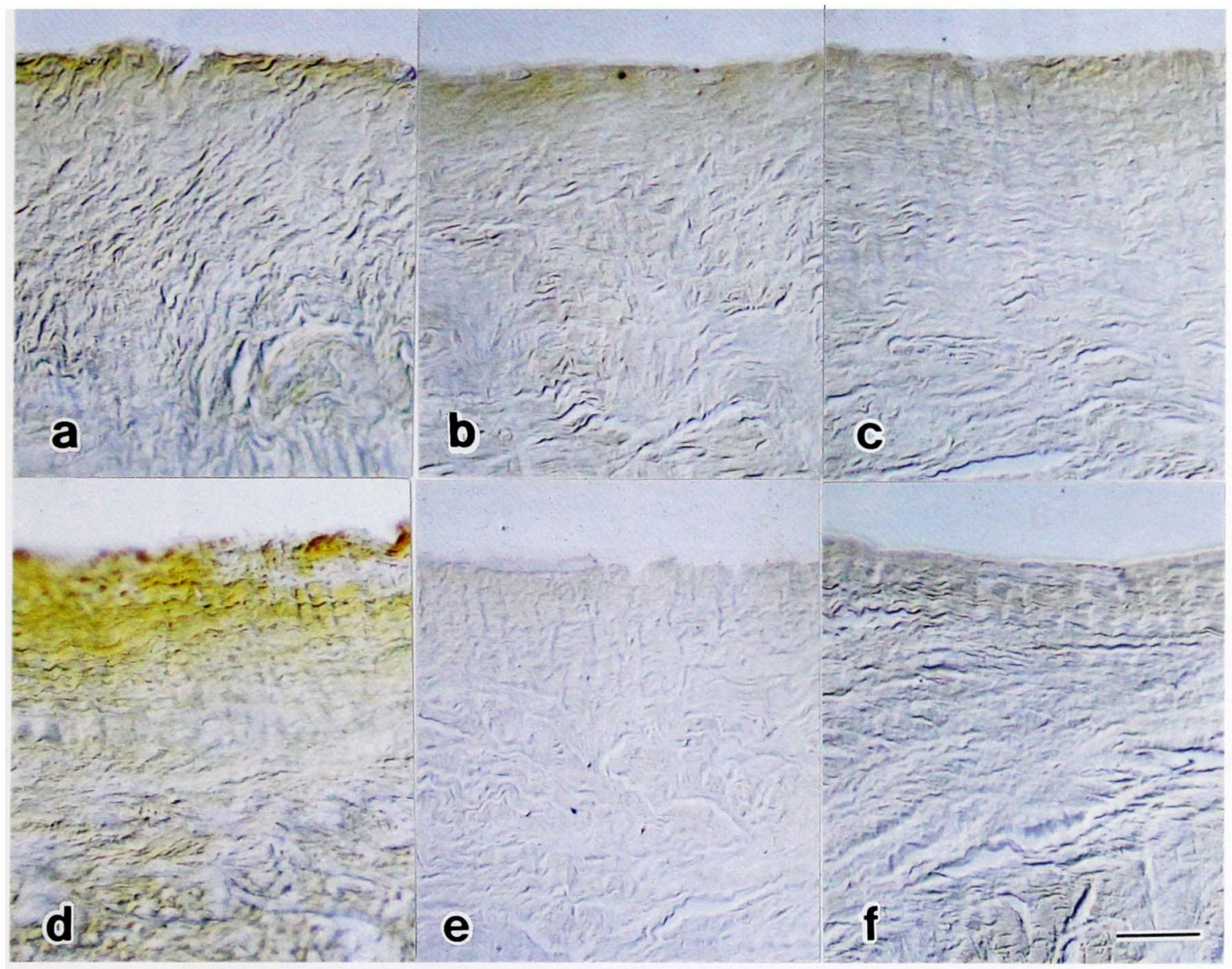

\section{Plate III}

Fig. 3. A high magnification of a frontal section of a human articular disk of the sternoclavicular joint subjected to immunohistochemical staining for collagen types I, III and V and proteins of the extracellular matrix (points 10, see Fig. 1). a: collagen type I; b: collagen type III; c: collagen type V; d: fibronectin; e: tenascin; f: laminin. Scale bar $=10 \mu \mathrm{m}$. 


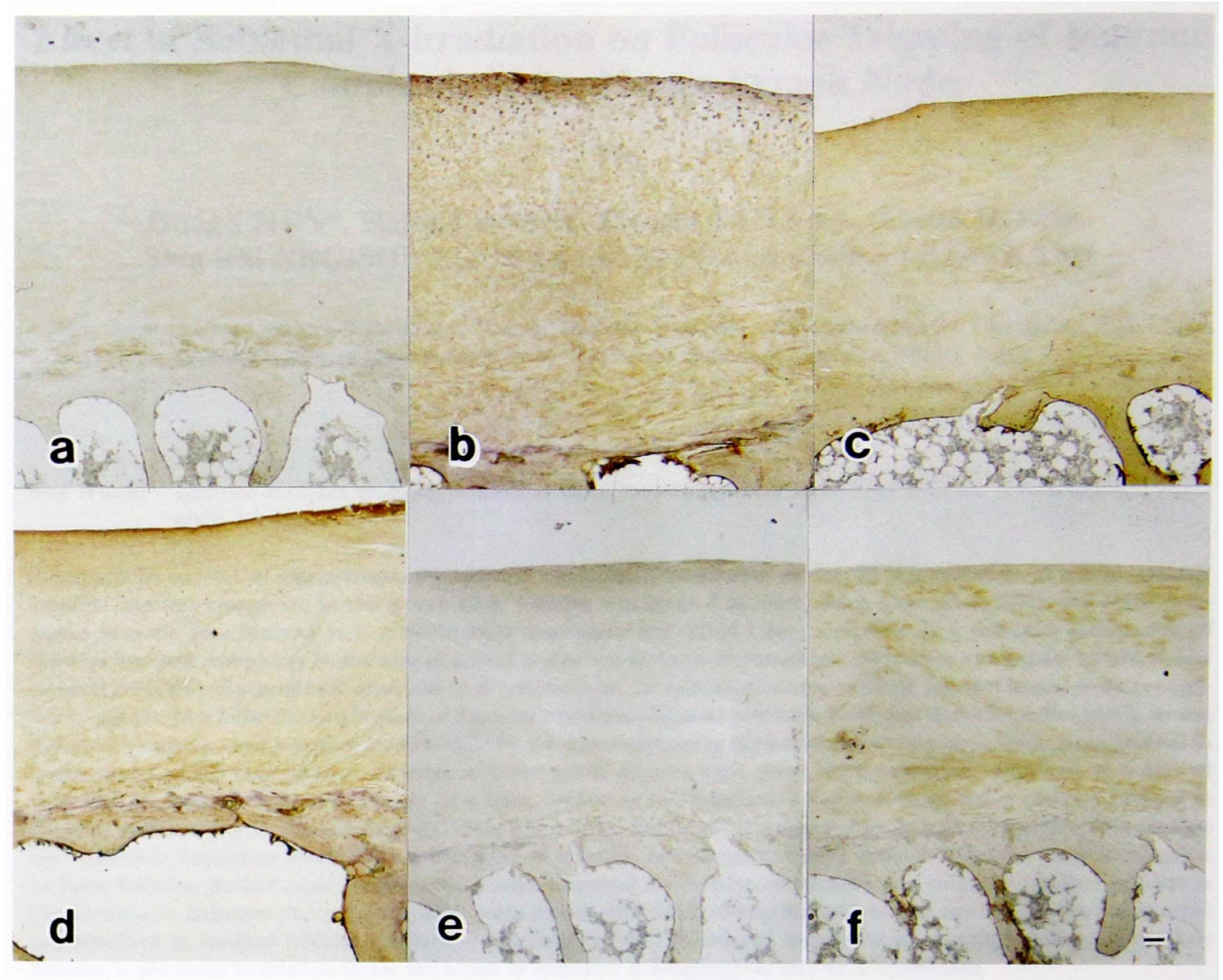

\section{Plate IV}

Fig. 4. A frontal section of the articular cartilage of the clavicle subjected to immunohistochemical staining for collagen type I, III and $V$ and proteins of the extracellular matrix (points 13, see Fig. 1). a: collagen type I; b: collagen type III; c: collagen type V; d: fibronectin; e: tenascin; f: laminin. Scale bar $=10 \mu \mathrm{m}$. 